\title{
Prophylactic use of a standardized botanical extract for the prevention of naturally occurring diarrhea in newborn Holstein calves
}

\author{
A. G. V. Teixeira, B. L. Ribeiro, P. R. M. Junior, H. C. Korzec, and R. C. Bicalho ${ }^{1}$ \\ Department of Population Medicine and Diagnostic Sciences, College of Veterinary Medicine, Cornell University, Ithaca, NY 14853-6401
}

\begin{abstract}
The objectives of this study were to evaluate the prophylactic use of SB-300 (Jaguar Animal Health, San Francisco, CA), a standardized botanical extract isolated from the bark latex of Croton lechleri, on reducing fecal water losses and diarrhea events in Holstein bull calves individually housed under a restricted whole-milk feeding regimen $(6 \mathrm{~L} / \mathrm{d})$ from 1 to $25 \mathrm{~d}$ of life. Fluid therapy administration due to dehydration, average weight gain, and the fecal microbiome were also evaluated. Bull calves used in this study were born from normal parturition, fed $4 \mathrm{~L}$ of pooled pasteurized colostrum by esophageal feeder, and moved to a research facility at Cornell University (Ithaca, NY). A doubleblinded randomized clinical trial was designed to allocate a total of 40 newborn calves into 1 of 2 treatment groups: calves receiving (twice daily) a solution containing $500 \mathrm{mg}$ of SB-300 added to the whole milk for the first $15 \mathrm{~d}$ of life (SB-300, $\mathrm{n}=20$ ) or a control group receiving sterile water added to whole milk for the same period (CTR, $\mathrm{n}=20)$. Treatment solutions had a total volume of $10 \mathrm{~mL}$ per treatment. Data regarding fecal dry matter were collected to precisely measure water content in fecal samples and to define diarrhea events; the SB-300 group had significantly increased fecal dry matter during the study period. Additionally, significantly fewer events of diarrhea were observed for calves in the SB-300 group (16.9\%) compared with calves in the CTR group (46.5\%). Dehydration status was evaluated and treated accordingly; calves with moderate dehydration were offered oral electrolytes, and calves with severe dehydration were rescued with intravenous fluid therapy. Calves in the SB-300 group had fewer intravenous fluid therapies administered during the study period $(1.6 \%)$ compared with the CTR group (3.1\%). Overall fluid therapy administered (oral electrolytes plus intravenous fluids) was significantly higher for the CTR group (9.2\%) compared with the SB-300 group (6.1\%) during the study period. No differences in milk
\end{abstract}

Received October 11, 2016.

Accepted December 18, 2016.

${ }^{1}$ Corresponding author: rcb28@cornell.edu consumption, calf starter intake, or weight gain were observed between treatment groups. A single time increase in Bifidobacterium was observed on d 20 of life for the SB-300 group; otherwise, no differences in fecal microbiome profile were detected between treatment groups. These results suggest that $500 \mathrm{mg}$ of SB-300 added to the milk for $15 \mathrm{~d}$ can reduce the incidence of diarrhea and reduce severe dehydration in milk-fed calves.

Key words: calves, diarrhea, microbiome, SB-300 botanical extract

\section{INTRODUCTION}

Neonatal calf diarrhea is a multifactorial disease that can be caused by infectious and noninfectious factors (Walker et al., 1998; O'Handley et al., 1999). In a report from the 2010 National Animal Health Monitoring System study, diarrhea was the most common disorder affecting preweaned dairy heifers, with a nationwide incidence of almost 19\%, and was the leading cause of death in preweaned heifers (USDA, 2012). Enteropathogens such as viruses, bacteria, and protozoa are often identified as etiological agents in calf diarrhea (Cho and Yoon, 2014).

Depending on the pathogen, calf diarrhea can have different pathophysiological mechanisms. These mechanisms have been well explained for Escherichia coli enterotoxin-mediated secretory diarrhea (Thiagarajah and Verkman, 2013), villus atrophy-mediated malabsorptive diarrhea caused by Cryptosporidium parvum (Heine et al., 1984), and coronavirus (Lewis and Phillips, 1978). Additionally, rotavirus can cause villus atrophy-mediated and chloride secretion-mediated diarrhea (Thiagarajah and Verkman, 2003). Briefly, the mechanisms by which loose stools are produced could be categorized as secretory, malabsorptive, or both.

Mixed infections are often reported in calves suffering from naturally occurring diarrhea (Reynolds et al., 1986). The prevalence of pathogens capable of producing secretory-induced neonatal diarrhea is reported to range between 2.0 and $45.0 \%$ for enterotoxigenic Escherichia coli (Frank and Kaneene, 1993; Luginbühl et al., 2005; Gulliksen et al., 2009) and between 17.0 and 
$80.0 \%$ for rotavirus (Bartels et al., 2010; Smith, 2012; Klein-Jöbstl et al., 2014). These pathogens can induce secretory diarrhea by increasing chloride secretion in the intestinal lumen, consequently leading to intestinal fluid hyper-secretion (Thiagarajah and Verkman, 2003).

Extensive review on prevention and treatment of neonatal calf diarrhea is available (Constable, 2009). Fluid therapy remains the standard treatment for undifferentiated naturally occurring neonatal calf diarrhea. However, approximately $55 \%$ of calf operations facilities in the United States make use of medicated milk replacers to control neonatal calf diarrhea, and about $54 \%$ of operations treat their scouring calves with antibiotics (Walker et al., 2012). Five antimicrobials are approved, labeled, and allowed by the US Food and Drug Administration to be used in medicated milk replacer for the control of bacteria; however, maximum effort should be made to avoid routine administration of antibiotics because of the risk of antimicrobial resistance (van den Bogaard and Stobberingh, 2000). Therefore, alternatives to antimicrobials for control and prevention of neonatal calf diarrhea are needed.

Recently, a natural product with antisecretory properties have shown efficacy in reducing water losses measured in fecal samples of neonatal Holstein bull calves experimentally challenged with enterotoxigenic E. coli (Teixeira et al., 2015). The natural product comprises proanthocyanidin oligomers, which are polyphenolic molecules extracted from the bark latex of the plant species Croton lecheri. This botanical extract has been studied for its antisecretory actions that involve the inhibition of 2 distinct chloride channels on the luminal membrane of the intestine: cystic fibrosis transmembrane conductance regulator (CFTR) and calciumactivated chloride channel (CaCC) (Fischer et al., 2004; Tradtrantip et al., 2010).

The objective of this study was to evaluate the effect of daily doses of a botanical extract on fecal water content, diarrhea incidence, and use of fluid therapy in Holstein bull calves affected with naturally occurring undifferentiated diarrhea. Additionally, we used a metagenomic approach to describe the effect of the botanical extract on the fecal microbiome. The study hypothesis was that fecal dry matter, diarrhea incidence, and use of fluid therapy are affected by the use of an anti-secretory botanical extract when given in milk.

\section{MATERIALS AND METHODS}

\section{Experimental Design, Animals, and Facility}

This experimental protocol was approved by the Institutional Animal Care and Use Committee (IACUC) of Cornell University (protocol number 2013-0075). Sample size was calculated based on a fecal DM difference previously evaluated by a pilot study. Based on an expected mean difference of fecal DM percentage between treatment groups of 1.4 , a treatment group standard deviation of 1.2, and a control group standard deviation of 1.7, with a treatment group ratio of 1 , and assuming a type I error rate of $5 \%$ and a power of $80 \%$, a sample size of 18 calves per group was calculated. As $10 \%$ mortality was anticipated, 20 calves per group were enrolled in this study.

The study design was a double-blinded randomized clinical trial. Randomization was performed a priori using the Excel random function (Microsoft Corp., Redmond, WA) to create a balanced number of calves per treatment group. A total of 40 Holstein bull calves from one commercial dairy farm (Scipio Center, NY) were enrolled in the study. Newborn Holstein bull calves were monitored during parturition by on farm staff. Calves were eligible to be enrolled in the study if no assistance was required during parturition, no twins, and no birth defects detected by physical exam after parturition. To mimic farm conditions, $4 \mathrm{~L}$ of pasteurized $\left(60^{\circ} \mathrm{C}\right.$ for 60 min) pooled colostrum (T-300 GoodNature Products Inc. Orchard Park, NY) with Brix reading averaging $22.8 \%$ (ranging from 20.6 to $24.8 \%$ ) was administered by esophageal tube to all calves within 45 min of birth (Oral Calf Feeder Bag with Probe, Jorvet, Loveland, $\mathrm{CO})$. All calves were transported from the farm to the study site within $4 \mathrm{~h}$ after birth. Briefly, calves were transported using an adapted vehicle for animal transportation; calves were kept inside individual crates with proper ventilation. Cleaning and sanitization of the vehicle was performed before and immediately after transportation.

The study site was a research barn with controlled temperature $\left(20.6^{\circ} \mathrm{C}\right.$, ranging from 19.4 to $\left.21.8^{\circ} \mathrm{C}\right)$ and humidity (50\%, ranging from 45 to $55 \%$ ) containing 20 individual stalls $\left(2.1 \mathrm{~m}^{2}\right.$ each $)$ isolated by concrete walls, where calves were unable to have any contact with other calves or outside areas. A 3-step cleaning procedure was used for water buckets, feed buckets, and bottles after each feeding, consisting of rinsing all the equipment with lukewarm water, scrubbing with a mixture of hot water and alkaline detergent solution, and finally rinsing in chlorinated water. The research barn used to conduct this trial was used twice because limited space was available (20 stalls). The first run was performed from January to March 2016, and the second run from March to May 2016. The 2 runs were performed using the same procedures for enrollment, data collection, cleaning, and laboratory procedures.

Calves were fed saleable whole milk purchased from the Cornell Teaching Dairy (Cornell University, Ithaca, 
NY) twice daily (0600 and $1800 \mathrm{~h}$ ) from first feeding to the end of study period at $25 \mathrm{~d}$ of life. From d 1, calves were gradually encouraged to drink from the bucket. Water was available ad libitum from d 1 until the end of the study. All calves were offered calf starter starting of d 16 of life (Calf Starter 18\% CP, DuMOR, Patterson, NY).

\section{Treatment Administration and Data Collection}

Randomization was performed in blocks (by dam parity and birth weight) a priori using the random function in Excel (Microsoft Corp.). Calves were randomly assigned into 1 of 2 treatment groups: control (CTR; n $=20)$ or standardized botanical extract $($ SB-300; $\mathrm{n}=$ 20; SB-300, Jaguar Animal Health, San Francisco, CA).

Treatment consisted of $500 \mathrm{mg}$ of SB-300 diluted in $10 \mathrm{~mL}$ of sterile water (VetOne, Boise, ID) using a conical twist-top sterile tube (Corning Falcon, Big Flats, NY). Treatments were prepared by adding 10 $\mathrm{mL}$ of sterile water and $500 \mathrm{mg}$ of SB-300 into a $15-\mathrm{mL}$ conical tube and placing it into a benchtop tube mixer at low speed for $1 \mathrm{~h}$ before each feeding (TubeRevolver, SoCal Biomedical, Orange County, CA). Treatments were administered during feeding by adding the solution into the milk, twice daily, for the first $15 \mathrm{~d}$ of life. Control calves were administered $10 \mathrm{~mL}$ of sterile water by adding the solution into the milk, twice daily, for the first $15 \mathrm{~d}$ of life.

To accomplish double-blindness, one member of the research team performed only randomization and calf stall assignment. Another member of the research team was responsible for morning and evening feedings and treatments (treatments were added to the milk at the time of feeding). A third member of the research team was responsible for collecting the data.

Calves were weighed at birth, 5, 10, 15, 20, and $25 \mathrm{~d}$ of life using a digital portable scale (Waypig-15, Vittetoe Inc., Keota, IA). Rectal fecal swabs for metagenomic data were also collected at birth, 5, 10, 15, 20, and $25 \mathrm{~d}$ of life using DNA-free sterile swabs (6" Sterile DNA-Free Cotton Swab, Puritan Medical Products Company LLC, Guilford, ME), placed into a $1.5-\mathrm{mL}$ sterile conical flask, identified with a number, and placed in a $-80^{\circ} \mathrm{C}$ freezer within $10 \mathrm{~min}$ of sampling. Blood samples were collected via jugular venipuncture using an 18-gauge $\times 3.8$-cm-long needle into $10-\mathrm{mL}$ Vacutainer tubes (Becton, Dickinson and Co., Franklin Lakes, NJ). Blood sampling was performed on d 2 of life. Serum was harvested following centrifugation at $2,000 \times g$ for 15 min at $4^{\circ} \mathrm{C}$. Serum IgG was measured using the second blood collection (d 2 of life) using a radial immunodiffusion assay according to kit instructions (Bethyl Laboratories Inc., Montgomery, TX).
All calves were examined twice daily within $2 \mathrm{~h}$ after feeding from d 1 to 25 . The following parameters were evaluated during each examination: rectal temperature (Digital Rectal Thermometer, McKesson, CA), recession of eyes into the orbit (distance between the medial canthus and the eyeball in millimeters), skin tent (thoracic pinch measuring skin flattening in seconds), attitude (suckling reflexes, standing position, and resting position), milk consumption, and calf starter intake (from d 15 to 25 of life). Additionally, fecal samples were collected via digital rectal stimulation twice daily within $1 \mathrm{~h}$ after feeding onto aluminum weighing boats from d 1 to 15 , on d 20, and a last collection performed once on d 25 of life and immediately used for DM evaluation. For all fecal samples collected, determination of fecal DM was performed as described by Bellosa et al. (2011). Briefly, samples were weighed on a precision digital scale (6202-1S, Sartorius, Elk Grove, IL) and then dried at $108^{\circ} \mathrm{C}$ for $24 \mathrm{~h}$ (model 10 Lab Oven, Quincy Lab, Chicago, IL) and re-weighed immediately to determine the percentage DM. To avoid subjectivity from visually scoring calves with diarrhea based on fecal consistency, diarrhea was defined after data on fecal DM were collected. An event of diarrhea was given to a calf that presented at least one of the twice-daily measurements of fecal DM $\leq 10.0 \%$.

A fixed amount of saleable whole milk was offered for the entire study period (3 L/feeding; $6 \mathrm{~L}$ total per day); milk consumption was measured after each meal from d 1 to the last day of the study. Calf starter was offered only after treatment cessation (on d 15 after second feeding); $4 \mathrm{~kg}$ of calf starter was offered and weighed again at $25 \mathrm{~d}$ of life, and average calf starter intake was calculated from d 16 to 25 by subtracting the calf starter offered final weight (d 25) by the initial weight (d 16) and dividing by 9 (9 d).

Dehydration was assessed twice daily and fluid therapy was administered accordingly. Briefly, calves presenting minor loss of skin elasticity (thoracic skin pinch $<3 \mathrm{~s})$ and eyes not recessed into orbit $(\leq 2 \mathrm{~mm})$ were considered not dehydrated and no rescue was performed. Calves with skin tent $>3$ s but $<10$ s, eyes slightly recessed into orbit $(>2 \mathrm{~mm}$ but $\leq 4 \mathrm{~mm}$ ), and with sucking reflexes were considered moderately dehydrated (needing $\leq 6 \%$ of $\mathrm{BW}$ in fluid replacement) and given oral fluid therapy (fixed volume, calculated as $6 \%$ multiplied by the most recently measured BW). Oral hydration was also performed with a commercial oral electrolyte (Hydralyte, Lloyd Inc., Shenandoah, IA); oral electrolyte was fed via bottle as an extra meal administered at time of physical exam. Calves with skin tent $>10 \mathrm{~s}$, eyes markedly recessed into orbit $(\geq 5$ $\mathrm{mm}$ ), with or without suckling reflexes were considered severely dehydrated (needing $\geq 8 \%$ of $\mathrm{BW}$ in fluid 
replacement) and given intravenous rescue therapy (fixed volume, calculated as $8 \%$ multiplied by the most recently measured BW). Intravenous fluid therapy using isotonic sodium bicarbonate was administered by jugular vein catheter $(70 \mathrm{~mL} / \mathrm{kg}$ per hour). During this study, no antimicrobials were used in addition to the fluid therapy.

\section{Next-Generation Sequencing Methodology}

Isolation of DNA from fecal contents was performed by adding the cotton head of each sample swab to 1.5 $\mathrm{mL}$ of nuclease-free water (Life Technologies, Grand Island, NY), and vortexing for $2 \mathrm{~min}$. The swab was then removed and the sample was centrifuged for $10 \mathrm{~min}$ at $13,200 \times g$. The supernatant was discarded and the remaining pellet was used for total metagenomic DNA extraction using the E.Z.N.A Stool DNA Kit (Omega Bio-Tek, Norcross, GA) following the manufacturer's guidelines. Concentration and purity of the DNA were evaluated by optical density using a spectrophotometer (NanoDrop Technologies, Rockland, DE), and approximately $350 \mathrm{ng}$ of DNA was used for PCR.

The 16S rRNA gene was amplified by PCR from individual metagenomic DNA samples from the fecal content using barcoded primers. For amplification of the V4 hypervariable region of the bacterial/archaeal $16 \mathrm{~S}$ rRNA gene, primers 515F and 806R were used according to previously described methods and optimized for the Illumina MiSeq platform (Illumina Inc., San Diego, CA; Caporaso et al., 2012). The earth microbiome project (www.earthmicrobiome.org) was used to select 280 different 12-bp barcodes for the 16S rRNA PCR, as previously described (Caporaso et al., 2012). The 5'-barcoded amplicons were generated in triplicate using 300 ng of template DNA, a master mix (Lucigen, Middleton, WI), and $10 \mu M$ each primer. The PCR conditions for the 16S rRNA gene included an initial denaturing step of $94^{\circ} \mathrm{C}$ for 3 min followed by 35 cycles of $94^{\circ} \mathrm{C}$ for $45 \mathrm{~s}, 50^{\circ} \mathrm{C}$ for $1 \mathrm{~min}$, and $72^{\circ} \mathrm{C}$ for $90 \mathrm{~s}$, and a final elongation step of $72^{\circ} \mathrm{C}$ for $10 \mathrm{~min}$. Replicate amplicons were pooled and purified with a QIAquick PCR Purification Kit (Qiagen, Valencia, CA) and visualized by electrophoresis through $1.2 \%$ (wt/vol) agarose gels stained with $0.5 \mathrm{mg} / \mathrm{mL}$ ethidium bromide before sequencing.

\section{Statistical Analyses}

Descriptive statistical analysis was undertaken in SAS (SAS Institute Inc., Cary, NC). Analysis of variance was used to evaluate differences in birth weight $(\mathrm{kg})$ at enrollment, serum IgG $(\mathrm{g} / \mathrm{L})$ collected at $2 \mathrm{~d}$ of life, and baseline fecal DM (\%). The intra- and interas- say coefficients of variation for serum IgG (quantified by an ELISA kit) were 3.0 and $3.8 \%$, respectively. Only 2 calves in the SB-300 group and 1 calf in the control group died. Briefly, all 3 calves presented with persistent fever (rectal temperature $>39.5^{\circ} \mathrm{C}$ ) from first feeding and depression (lost appetite, recumbency); they were diagnosed with septicemia and treated with antibiotics, corticosteroids, and intravenous fluids. Treatment was unsuccessful and euthanasia was performed. Data from these calves were excluded from all statistical analysis. Differences in mortality was evaluated by Fisher`s exact test using the FREQ procedure in SAS.

To evaluate the effect of treatment on daily milk intake, daily calf starter intake, and ADG, 5 general linear models were fitted to the data using the GLM procedure of SAS. The independent variables offered to the models were treatment group (CTR and SB-300), serum IgG at $2 \mathrm{~d}$ of life $(\mathrm{g} / \mathrm{L}), \mathrm{BW}$ at enrollment $(\mathrm{kg})$, and baseline fecal DM (\%). Treatment group was the only variable forced into the models and a backward stepwise variable selection was performed. The assumption that the residuals were normally distributed was assessed by visually evaluating the distribution plot of the Studentized residuals.

To evaluate the effect of treatment on fluid therapy (oral electrolyte, intravenous fluid, and overall fluid therapy), 3 multivariable mixed logistic regression models were fit to the data using the GLIMMIX procedure of SAS. The independent variables offered to the model were treatment group (CTR and SB-300), serum IgG $(\mathrm{g} / \mathrm{L}), \mathrm{BW}$ at enrollment $(\mathrm{kg})$, and baseline fecal DM (\%). Odds ratio and adjusted probabilities of diarrhea were obtained using the LSMEANS statement.

A mixed general linear mixed model was fitted to the data using the MIXED procedure of SAS to analyze the effect of treatment on fecal DM (\%). The independent variables offered to the model were treatment group (CTR and SB-300), serum IgG (g/L), BW at enrollment $(\mathrm{kg})$, and baseline fecal DM (\%). Fecal DM data were longitudinally collected and consisted of a total of 33 measurements per calf: twice daily from d 1 to 15 , twice daily on d 20, and once on d 25 of life before the end of the study. Therefore, data points were correlated within each research subject. To account appropriately for within-calf correlation, the error term was modeled by imposing a first-order autoregressive covariance structure. For this model, a backward stepwise variable selection was performed. Furthermore, to assess the effect of treatment on fecal DM collected during the study period, the interaction between treatment and days was forced into the model. Visual evaluation of the distribution plot of the Studentized residuals allowed us to assume that the residuals were normally distributed. 
Table 1. Description of newborn Holstein bull calves fed pasteurized pooled colostrum, individually housed, fed restricted whole milk (6 L/d) from d 1 to 25 of life, and offered calf starter from d 16 to 25 of life ${ }^{1}$

\begin{tabular}{|c|c|c|c|c|c|}
\hline \multirow[b]{2}{*}{ Item } & \multicolumn{2}{|c|}{ Control $(\mathrm{n}=20)$} & \multicolumn{2}{|c|}{ SB-300 $(\mathrm{n}=20)$} & \multirow[b]{2}{*}{$P$-value } \\
\hline & Mean & $95 \%$ CI & Mean & $95 \%$ CI & \\
\hline Mortality (\%) & 5 & & 10 & & 0.38 \\
\hline Birth weight (kg) & 37.6 & $37.3-37.9$ & 37.7 & $37.4-38.4$ & 0.57 \\
\hline Serum IgG $(g / L)$ & 12.3 & $11.6-13.0$ & 12.1 & $11.4-12.8$ & 0.72 \\
\hline Fecal DM, baseline (\%) & 29.8 & $23.5-36.1$ & 27.2 & $20.9-33.5$ & 0.55 \\
\hline
\end{tabular}

${ }^{1}$ Control calves were administered $10 \mathrm{~mL}$ of sterile water (added to milk), twice daily, for the first $15 \mathrm{~d}$ of life; calves in the SB-300 group were fed $500 \mathrm{mg}$ of a standardized botanical extract (SB-300, Jaguar Animal Health, San Francisco, CA) diluted in $10 \mathrm{~mL}$ of sterile water.

Data regarding events of diarrhea was created for each day from d 1 to 15 , on d 20 , and on d 25 of life. To evaluate the effect of treatment on diarrhea (yes or no), a multivariable mixed logistic regression model was fitted to the data using the GLIMMIX procedure of SAS. The independent variables offered to the model were treatment group (CTR and SB-300), serum IgG $(\mathrm{g} / \mathrm{L}), \mathrm{BW}$ at enrollment $(\mathrm{kg})$, and baseline fecal DM (\%). Adjusted probability of diarrhea was obtained using the LSMEANS statement.

The effect of treatment on BW collected at 1, 5, 10, 15,20 , and $25 \mathrm{~d}$ of life was evaluated by a mixed general linear model using the MIXED procedure of SAS. To control for repeated measures of BW, the animal identification number (nested within run) was included in all models as a random effect, and the independent variables offered to the model were treatment group (CTR and SB-300), serum IgG (g/L), BW at enrollment (kg), and baseline fecal DM (\%). For this model, a backward stepwise variable selection was performed and to assess the effect of treatment on fecal DM collected during the study period, the interaction between treatment and days was forced into the model. The assumption that the residuals were normally distributed was assessed by visually assessing the Studentized residuals plot.

Abundance of the individual genera Bifidobacterium, Lactobacillus, Fecalibacterium, and Escherichia were evaluated using 4 similar general linear mixed models in SAS. Variables offered to the models included treatment group (CTR and SB-300), days $(1,5,10,15,20$, and $25 \mathrm{~d}$ of life), and their interaction terms. To account appropriately for within-calf correlation, the error term was modeled by imposing a first-order autoregressive covariance structure.

\section{RESULTS}

Forty newborn Holstein bull calves were enrolled in this study. No differences in mortality was observed: 2 calves in the SB-300 group and 1 calf in the CTR group $(P=0.38)$ were euthanized due to septicemia. Birth weight did not differ between CTR $(37.6 \mathrm{~kg}, 95 \% \mathrm{CI}$ : 37.4-38.4) and SB-300 (37.7 kg, 95\% CI: 37.4-38.4). Serum IgG quantification was performed on d 2 of life, and no differences were found between calves in the CTR group (12.3 g/L, 95\% CI: 11.6-13.0) and SB-300 group (12.1 g/L, 95\% CI: 11.4-12.8). Additionally, no differences were found for baseline fecal DM between treatment groups $(P=0.55$, Table 1$)$.

\section{Milk Intake, Calf Starter Intake, and ADG}

No differences were observed for milk and calf starter intake between treatment groups (Table 2). Briefly, calves in the CTR group had an average milk intake of $5.38 \mathrm{~L} / \mathrm{d}$ (95\% CI: 5.11-5.65) and calves in the SB-300 group had an intake of $5.55 \mathrm{~L} / \mathrm{d}$ (95\% CI: 5.28-5.83). Calf starter was only offered after cessation of treatment at d 16 of life. Calf starter intake was not significantly different between treatment groups $(P=0.69)$. Moreover, no differences in ADG were observed from 1 to $25 \mathrm{~d}$ of life; calves in the CTR group had an ADG of $0.38 \mathrm{~kg} / \mathrm{d}$ (95\% CI: $0.27-0.49)$ and calves in the SB-300 group had an ADG of $0.43 \mathrm{~kg} / \mathrm{d}$ (95\% CI: $0.30-0.55)$.

\section{Dehydration and Fluid Therapy}

During the study period, no differences were observed between treatment groups when evaluating the odds of a calf being moderately dehydrated and needing oral electrolytes (odds ratio $=0.07, P=0.09$, Table 3 ) with an adjusted probability of $4.5 \%$ for calves in the SB-300 and $6.3 \%$ for calves in the CTR group. The odds of a calf being severely dehydrated and consequently needing intravenous fluids was 0.5 lower for calves in the SB-300 group than for calves in the CTR group $(P=$ 0.04 ), with an adjusted probability of $1.6 \%$ for calves in the SB-300 and $3.1 \%$ for calves in the CTR group. Overall fluid therapy administered (oral electrolyte plus intravenous fluid) was significantly higher for the CTR group (9.2\%) than for the SB-300 group (6.1\%) during the study period. Likewise, the odds of needing 
Table 2. The effect of treatment on milk consumption from d 1 to 25 of life, calf starter intake from d 16 to 25 of life, and the effect of treatment on ADG (in 3 periods; d 1 to 15 , d 15 to 25 , and d 1 to 25 of life) were evaluated by 5 different general linear mixed models ${ }^{1}$

\begin{tabular}{|c|c|c|c|c|c|c|}
\hline \multirow[b]{2}{*}{ Item } & \multirow[b]{2}{*}{ Period (d) } & \multicolumn{2}{|c|}{ Control $(\mathrm{n}=19)$} & \multicolumn{2}{|c|}{ SB-300 (n = 18) } & \multirow[b]{2}{*}{$P$-value } \\
\hline & & Mean & $95 \%$ CI & Mean & $95 \%$ CI & \\
\hline \multirow[t]{2}{*}{ Calf starter intake $(\mathrm{kg} / \mathrm{d})$} & 15 to 25 & 0.16 & $0.15-0.17$ & 0.16 & $0.15-0.17$ & 0.69 \\
\hline & 1 to 15 & 0.14 & $0.12-0.18$ & 0.17 & $0.14-0.21$ & 0.22 \\
\hline \multirow[t]{2}{*}{ Weight gain $(\mathrm{kg} / \mathrm{d})$} & 15 to 25 & 0.74 & $0.56-0.92$ & 0.89 & $0.71-1.08$ & 0.22 \\
\hline & 1 to 25 & 0.38 & $0.29-0.47$ & 0.46 & $0.37-0.56$ & 0.22 \\
\hline
\end{tabular}

${ }^{1}$ Control calves were administered $10 \mathrm{~mL}$ of sterile water (added to milk), twice daily, for the first $15 \mathrm{~d}$ of life; calves in the SB-300 group were fed $500 \mathrm{mg}$ of a standardized botanical extract (SB-300, Jaguar Animal Health, San Francisco, CA) diluted in $10 \mathrm{~mL}$ of sterile water.

any fluid therapy (due to moderate or severe dehydration) during the study period were 0.6 times lower in the SB-300 group than in the CTR group.

\section{Fecal DM and Diarrhea}

A significantly higher fecal DM percentage was observed for calves in the SB-300 group $(18.2 \%, 95 \% \mathrm{CI}$ : $17.2-19.2)$ than for calves in the CTR group $(13.2 \%$, $95 \%$ CI: $12.2-14.2)$ during the study period $(P<$ 0.0001 , Figure 1). Likewise, when evaluating the effect of treatment on events of diarrhea, calves in the SB-300 group had significantly fewer events of diarrhea (16.9\%, 95\% CI: 12.4-22.5) than calves in the CTR group $(46.5 \%, 95 \%$ CI: 39.9-53.2) during the study period $(P<0.0001$, Figure 2$)$. For both outcomes, the interaction term between treatment group and days of life was not significant and was forced in the models to generate daily estimates for both fecal DM $(P=0.22)$ and diarrhea $(P=0.75)$.

\section{$B W$}

No effect of treatment was observed for BW data collected during the study period $(P=0.42$, Figure
3). The interaction term between treatment group and days of life was not significant and was forced into the model $(P=0.14)$. Briefly, BW by the end of the study period ( $25 \mathrm{~d}$ of life) was $49.2 \mathrm{~kg}$ (95\% CI: 47.7-50.6) for calves in the SB-300 group and 47.0 (95\% CI: 45.6-48.5) for calves in the CTR group.

\section{Fecal Microbiome}

The 3 most abundant phyla described in this study were Firmicutes, Proteobacteria, and Bacteroidetes, regardless of treatment group. No differences in mean relative abundance of each phylum within days of life between treatment groups were observed (Figure 4). To investigate beneficial genera of intestinal bacteria, an individual evaluation of the following bacterial genera was performed: Bifidobacterium (Vlková et al., 2006; Bunesova et al., 2012), Lactobacillus (Ewaschuk et al., 2004), and Fecalibacterium (Oikonomou et al., 2013; Foditsch et al., 2015). Additionally, the Escherichia genus was considered for its known association with unhealthy microbiome (Acres, 1985). Bifidobacterium had a higher mean relative abundance at d 20 for SB300 calves compared with CTR calves $(P<0.05)$. However, that was the only difference observed from data

Table 3. Dehydration was assessed twice daily for all calves in the study from d 1 to 25 of life; calves with moderate dehydration were offered oral electrolytes and calves with severe dehydration were rescued with intravenous fluid therapy ${ }^{1,2}$

\begin{tabular}{|c|c|c|c|c|}
\hline \multirow[b]{2}{*}{ Item } & \multicolumn{2}{|c|}{$\begin{array}{c}\text { Adjusted } \\
\text { probability (\%) }\end{array}$} & \multirow{2}{*}{$\begin{array}{l}\text { Odds ratio } \\
(95 \% \mathrm{CI})\end{array}$} & \multirow[b]{2}{*}{$P$-value } \\
\hline & Control & SB-300 & & \\
\hline Oral electrolytes & 6.3 & 4.5 & $0.7(0.5-1.0)$ & 0.09 \\
\hline Intravenous fluids & 3.1 & 1.6 & $0.5(0.3-0.9)$ & 0.04 \\
\hline Overall fluid therapy & 9.2 & 6.1 & $0.6(0.4-0.9)$ & 0.01 \\
\hline
\end{tabular}

${ }^{1}$ The effect of treatment on fluid therapy was evaluated by 3 generalized linear mixed models. Calves in the control group were used as a reference level.

${ }^{2}$ Control calves were administered $10 \mathrm{~mL}$ of sterile water (added to milk), twice daily, for the first $15 \mathrm{~d}$ of life; calves in the SB-300 group were fed $500 \mathrm{mg}$ of a standardized botanical extract (SB-300, Jaguar Animal Health, San Francisco, CA) diluted in $10 \mathrm{~mL}$ of sterile water. 


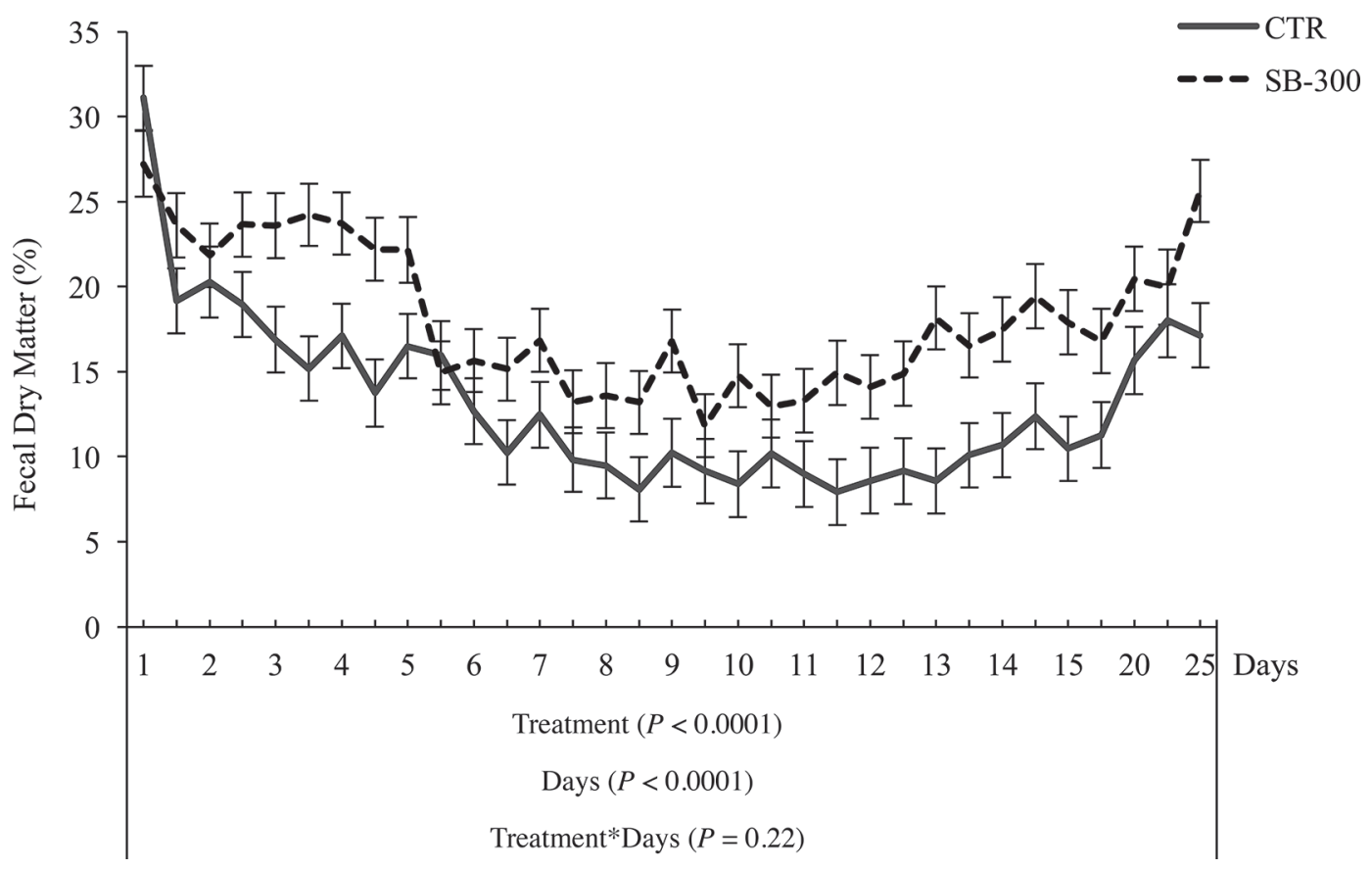

Figure 1. Fecal DM was measured twice daily for each of the treatment days (d 1 to 15). Additional samples were collected twice on d 20 and once on d 25 before the end of the study. Treatments were administered twice daily with whole milk for the first $15 \mathrm{~d}$ of life. The effect of treatment, days, and the interaction between treatment and days are also displayed. Control calves $(\mathrm{n}=19)$ were administered $10 \mathrm{~mL}$ of sterile water (added to milk), twice daily, for the first $15 \mathrm{~d}$ of life; calves in the SB-300 group ( $\mathrm{n}=18$ ) were fed $500 \mathrm{mg}$ of a standardized botanical extract (SB-300, Jaguar Animal Health, San Francisco, CA) diluted in $10 \mathrm{~mL}$ of sterile water. Values are least square means \pm standard errors.

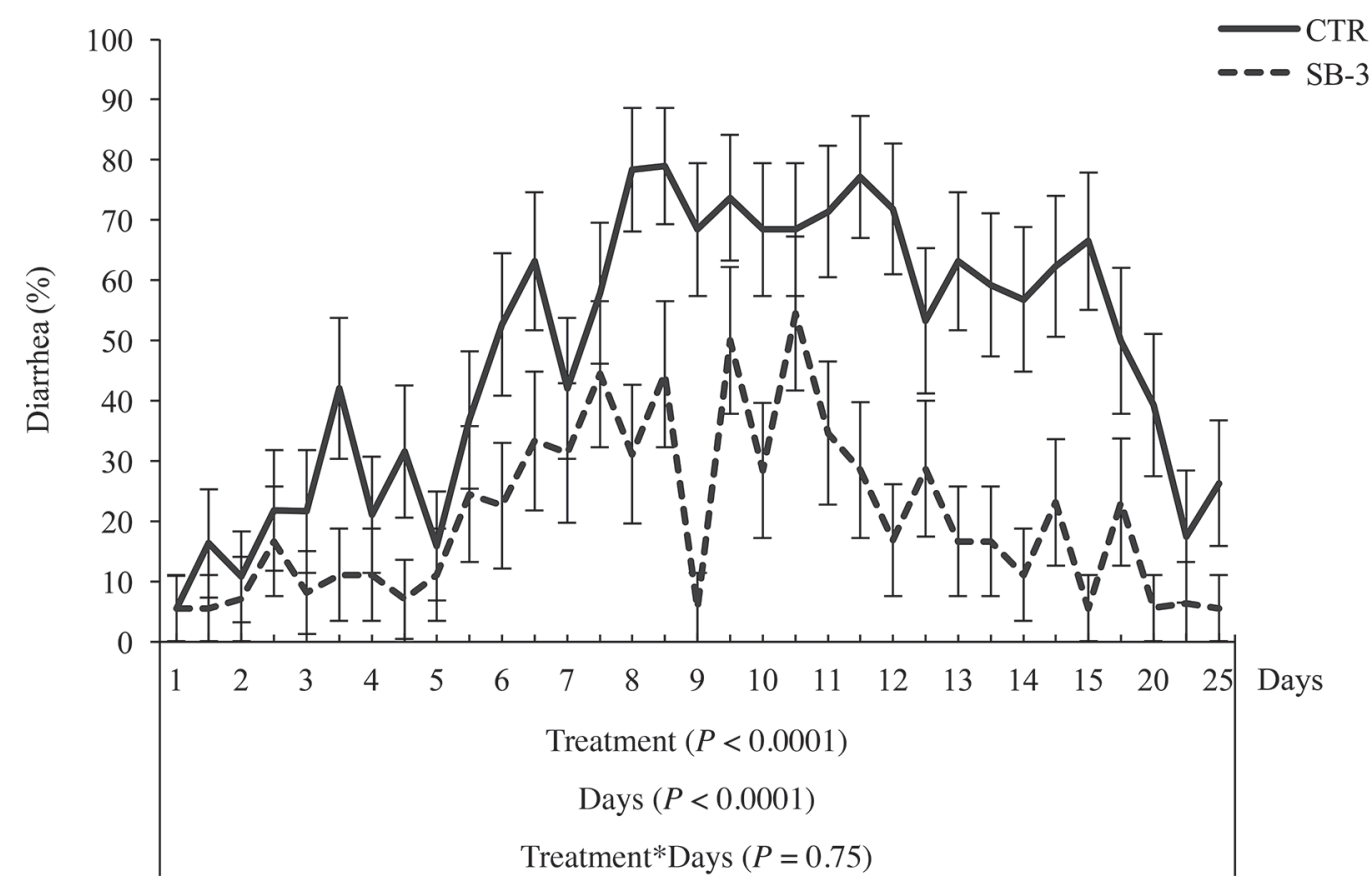

Figure 2. Diarrhea was recorded every day for all the calves in the study; an event of diarrhea was confirmed when a calf fecal sample presented at least one of the twice-daily measurements of fecal DM $\leq 10.0 \%$. Treatments were administered twice daily with whole milk for the first $15 \mathrm{~d}$ of life. The effect of treatment, days, and the interaction between treatment and days are also displayed. Control calves $(\mathrm{n}=19) \mathrm{were}$ administered $10 \mathrm{~mL}$ of sterile water (added to milk), twice daily, for the first $15 \mathrm{~d}$ of life; calves in the SB-300 group (n = 18) were fed $500 \mathrm{mg}$ of a standardized botanical extract (SB-300, Jaguar Animal Health, San Francisco, CA) diluted in 10 mL of sterile water. The y-axis represents the model-adjusted proportion of calves with diarrhea and $\mathrm{x}$-axis represents days into the study. Values are least square means \pm standard errors. 


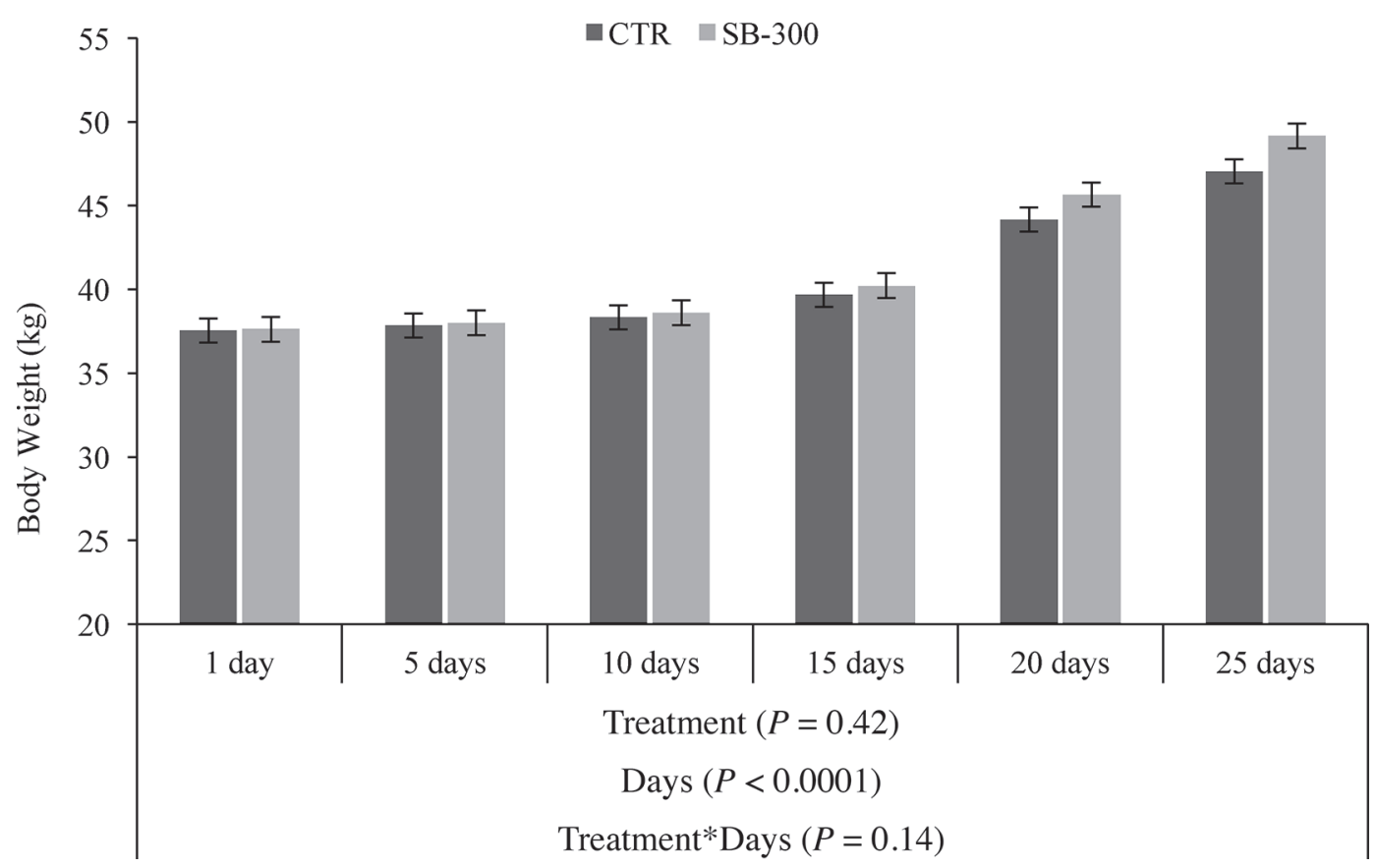

Figure 3. The effect of treatment on BW measured at 5, 10, 15, 20 and $25 \mathrm{~d}$ of life was evaluated by a general linear mixed model. The effect of treatment, days, and the interaction between treatment and days are displayed. The y-axis represents the least squares means of BW and the $\mathrm{x}$-axis represents the days of $\mathrm{BW}$ measurement. Control calves (CTR; $\mathrm{n}=19$ ) were administered $10 \mathrm{~mL}$ of sterile water (added to milk), twice daily, for the first $15 \mathrm{~d}$ of life; calves in the SB-300 group ( $\mathrm{n}=18)$ were fed $500 \mathrm{mg}$ of a standardized botanical extract (SB-300, Jaguar Animal Health, San Francisco, CA) diluted in $10 \mathrm{~mL}$ of sterile water. Values are least square means \pm standard errors.

on genera; no differences were found in mean relative abundance of any genera between treatment groups (Figure 5).

\section{DISCUSSION}

This double-blinded randomized clinical trial evaluated the prophylactic use of a standardized botanical extract administered twice daily at feeding mixed with saleable whole milk for the first $15 \mathrm{~d}$ of life on naturally occurring diarrhea. Diarrhea was precisely defined by measuring DM content in fecal samples collected twice daily from d 1 to 15 , on d 20, and on d 25 of life. The main findings of this study were the decreased incidence of undifferentiated naturally occurring diarrhea (defined as fecal $\mathrm{DM} \leq 10.0 \%$ ) and fewer administrations of fluid therapy (due to severe and moderate dehydration) in Holstein bull calves housed individually and fed a restricted amount of saleable whole milk for $25 \mathrm{~d}$. We detected no differences in milk consumption or ADG. No effect of treatment on the intestinal microbiome was observed, besides a transitory increase for the genus Bifidobacterium at $20 \mathrm{~d}$ of life for calves in the SB-300 group compared with control calves.

Previously, when calves were challenged with enterotoxigenic Escherichia coli, the antisecretory properties of the standardized botanical extract in a secretoryinduced diarrhea set-up significantly decreased water content in fecal samples (Teixeira et al., 2015). In the current study, a twice-daily dose of $500 \mathrm{mg}$ per feeding (3 L of whole saleable milk) of SB-300 for the first $15 \mathrm{~d}$ of life was able to increase DM content in fecal samples and consequently reduce the incidence of diarrhea. The diarrhea affecting calves in this trial can be considered undifferentiated naturally occurring diarrhea, because no attempt was made to identify the cause of diarrhea.

During an event of secretory diarrhea, a calf can lose significant amount of body fluids and, with it, blood electrolytes (Dalton et al., 1965; Phillips et al., 1971; Fisher and De la Fuente, 1972). Assessing dehydration and accurately identifying a calf that requires fluid therapy is very important. Dehydration can be successfully treated and provide calves with the necessary electrolytes and nutrients to restore its hydration status (Groutides and Michell, 1990; Hartmann and Reder, 1995; Sen et al., 2009; Smith, 2009). As previously described in calves with induced dehydration and diarrhea, the best clinical predictors of dehydration in neonatal calves are the degree of enophthalmos and neck skin-tent duration (Constable et al., 1998).

In the current trial, degree of dehydration was assessed by both described methods, and fluid therapy 
was administered accordingly. Oral electrolytes were administered when a calf was found to have moderate dehydration; no differences were detected between treatment groups. However, significantly more intravenous fluid treatments due to severe dehydration were administered to control calves than to SB-300 calves. Finally, overall fluid therapy administration (oral and intravenous fluids) was significantly different between treatment groups. In this study, calves suffering from undifferentiated diarrhea could have benefited from a constant dose of the standardized botanic extract offered twice daily for $15 \mathrm{~d}$; as reported by Phillips et al. (1971), neonatal calves suffering from secretory diarrhea can lose up to $71.4 \%$ of water in feces, and reducing this intestinal water secretion can reduce the severity of dehydration.

In this study, calves were offered a restricted amount of milk per day ( $6 \mathrm{~L})$ from d 1 to 25 of life, and calf starter was offered only after treatment ceased, starting at d 16 until d 25. Milk was offered even if a calf was under fluid therapy; as reported by others, calves suffering from diarrhea would benefit from the energy provided by the milk offered during feeding (Heath et al., 1989; Garthwaite et al., 1994). No differences were found between daily milk consumption and starter intake, and no effects on ADG were detected. In a multiherd study evaluating risk factors that could impair calf growth, neonatal calf diarrhea was one of the risk factors associated with lesser BW gain (Virtala et al., 1996; Windeyer et al., 2014). In the current study, BW did not differ significantly between treatment groups during the study period.

Here, we investigated the effect of a botanical extract on the fecal microbiota using a metagenomic approach. In this study, the hypothesis behind the microbiome investigation was that lesser secretion of water during an event of secretory diarrhea would lead to a more stable microbiome, possibly favoring beneficial bacteria that could lead to improved performance. During events of diarrhea triggered by E. coli, rotavirus, and coronavirus, the intestinal lumen can create a favorable environment for overgrowth of pathogenic bacteria (Tzipori et al., 1981, 1983; Kaske, 1993). The neonatal calf intestinal microbiota is dynamic during the first week of life, and different intestinal microbiota profiles can be associated with disease and weight gain (Uyeno

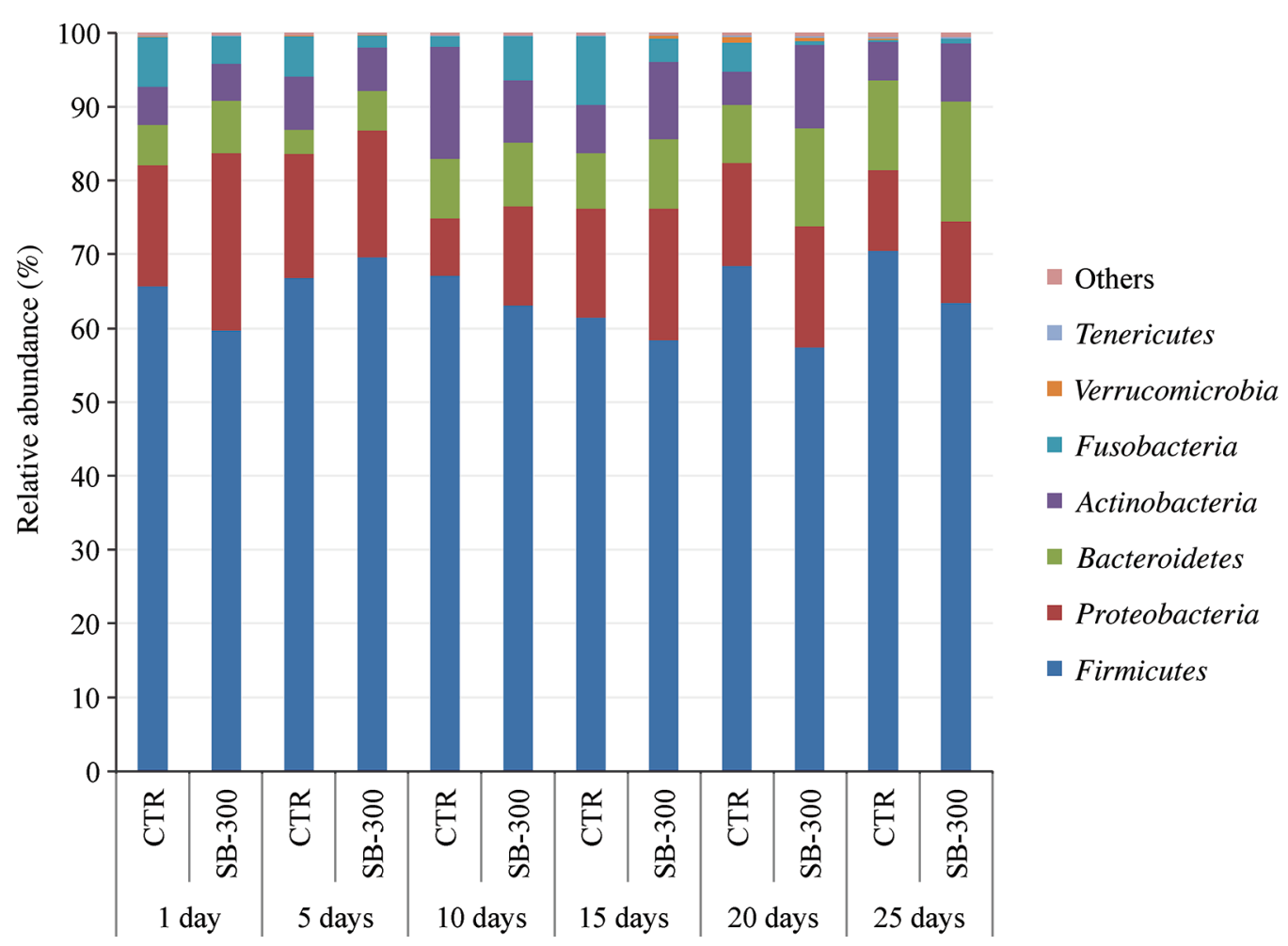

Figure 4. Descriptive distribution of the mean relative abundance (\%) of the most prevalent bacterial phyla identified in fecal samples of control calves (CTR, $\mathrm{n}=19$ ) and calves receiving standardized botanical extract (SB-300, $\mathrm{n}=18$ ). Control calves were administered $10 \mathrm{~mL}$ of sterile water (added to milk), twice daily, for the first $15 \mathrm{~d}$ of life; calves in the SB-300 group were fed 500 mg of a standardized botanical extract (SB-300, Jaguar Animal Health, San Francisco, CA) diluted in $10 \mathrm{~mL}$ of sterile water. Fecal samples were collected once daily at 1, 5, 10, 15, 20 , and $25 \mathrm{~d}$ of life. Color version available online. 

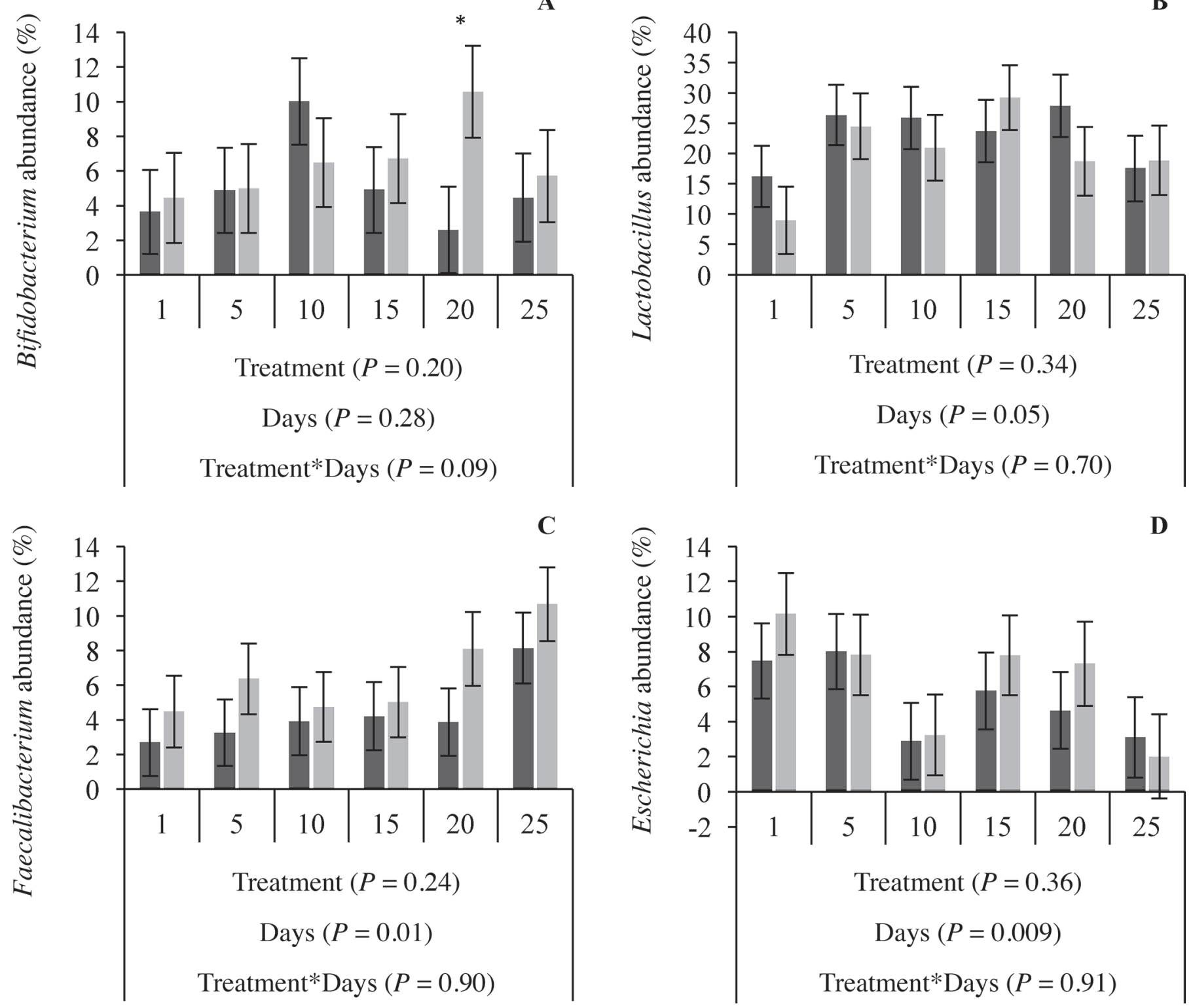

Figure 5. Comparison of mean relative abundance of 3 genera previously associated with healthy gut microbiome: Bifidobacterium (A), Lactobacillus (B), Fecalibacterium (C), and 1 genus related to unhealthy microbiota: Escherichia (D) found in fecal samples. Control calves (dark gray bars; $\mathrm{n}=19$ ) were administered $10 \mathrm{~mL}$ of sterile water (added to milk), twice daily, for the first $15 \mathrm{~d}$ of life; calves in the SB-300 group (light gray bars; $\mathrm{n}=18$ ) were fed $500 \mathrm{mg}$ of a standardized botanical extract (SB-300, Jaguar Animal Health, San Francisco, CA) diluted in $10 \mathrm{~mL}$ of sterile water. The $\mathrm{y}$-axis represents the mean relative abundance in percentage and the $\mathrm{x}$-axis represents the data point of each fecal sample collected (days of sampling). Values are least square means \pm standard errors. ${ }^{*} P<0.003$ : statistical difference adjusted by Bonferroni between CTR and SB-300 within day.

et al., 2010; Oikonomou et al., 2013; Malmuthuge et al., 2015). In neonatal calves, the beneficial effects of decreasing diarrhea incidence and improving weight gain in calves have been reported when a higher prevalence of certain bacterial genera exists; namely Lactobacillus, Bifidobacterium, and Fecalibacterium (Abe et al., 1995; Foditsch et al., 2015). In the current study, fecal swabs were collected during treatment days for metagenomic sequencing, and no differences in relative abundance between treatment groups were detected for the genera Lactobacillus, Bifidobacterium, and Fecalibacterium. Fecal swabs were also collected on d 20 and 25; a difference was observed on d 20 only for Bifidobacterium between treatment groups, where calves in the SB-300 
group had a higher relative abundance of Bifidobacterium than calves in the control group. However, by d 25 , no differences were reported.

\section{CONCLUSIONS}

Forty newborn Holstein bull calves fed pasteurized pooled colostrum were raised under restricted feeding of saleable whole milk, housed individually from d 1 to 25 of life. A prophylactic dose of $500 \mathrm{mg}$ of a standardized botanical extract (SB-300) was added to the milk twice daily at feeding for $15 \mathrm{~d}$, and diarrhea events were precisely identified by percentage of water in the feces. Calves in the SB-300 treatment group experienced significantly fewer events of diarrhea during the study period (16.9\%) compared with control calves (46.5\%). Control calves suffered more from severe dehydration and had more intravenous fluid therapy interventions. No effects of treatment on average milk intake, calf starter intake, or ADG were observed. Besides a single increase in Bifidobacterium observed at d 20 for the SB-300 group, no differences in fecal microbiome profile were detected between treatment groups. Combined, these results suggest that $500 \mathrm{mg}$ of SB-300 added to the milk during feeding for $15 \mathrm{~d}$ can reduce the incidence of diarrhea and reduce severe dehydration in milk-fed calves.

\section{ACKNOWLEDGMENTS}

Jaguar Animal Health (San Francisco, CA) manufactured the evaluated botanic extract and funded the research presented herein. The authors acknowledge the ethnomedical expertise of the indigenous peoples of the Northwestern Amazon region who discovered how to use the latex of Croton lechleri for the treatment of diarrhea and other gastrointestinal conditions. Jaguar Animal Health played no role in the study design nor in the collection, analysis, interpretation of data, nor in the decision to submit the manuscript for publication. None of the authors has any financial or personal relationships that could inappropriately influence or bias the content of the paper.

\section{REFERENCES}

Abe, F., N. Ishibashi, and S. Shimamura. 1995. Effect of administration of bifidobacteria and lactic acid bacteria to newborn calves and piglets. J. Dairy Sci. 78:2838-2846. https://doi.org/10.3168/ jds.S0022-0302(95)76914-4.

Acres, S. D. 1985. Enterotoxigenic Escherichia coli infections in newborn calves: A review. J. Dairy Sci. 68:229-256.

Bartels, C. J., M. Holzhauer, R. Jorritsma, W. A. Swart, and T. J. Lam. 2010. Prevalence, prediction and risk factors of enteropathogens in normal and non-normal faeces of young
Dutch dairy calves. Prev. Vet. Med. 93:162-169. https://doi. org/10.1016/j.prevetmed.2009.09.020.

Bellosa, M. L., D. V. Nydam, J. L. Liotta, J. A. Zambriski, T. C. Linden, and D. D. Bowman. 2011. A comparison of fecal percent dry matter and number of cryptosporidium parvum oocysts shed to observational fecal consistency scoring in dairy calves. J. Parasitol. 97:349-351.

Bunesova, V., K. J. Domig, J. Killer, E. Vlková, J. Kopecny, J. Mrazek, S. Rockova, and V. Rada. 2012. Characterization of bifidobacteria suitable for probiotic use in calves. Anaerobe 18:166-168. https:// doi.org/10.1016/j.anaerobe.2011.09.008.

Caporaso, J. G., C. L. Lauber, W. A. Walters, D. Berg-Lyons, J. Huntley, N. Fierer, S. M. Owens, J. Betley, L. Fraser, M. Bauer, N. Gormley, J. A. Gilbert, G. Smith, and R. Knight. 2012. Ultrahigh-throughput microbial community analysis on the Illumina HiSeq and MiSeq platforms. ISME J. 6:1621-1624.

Cho, Y.-I., and K.-J. Yoon. 2014. An overview of calf diarrhea-Infectious etiology, diagnosis, and intervention. J. Vet. Sci. 15:1-17. https://doi.org/10.4142/jvs.2014.15.1.1.

Constable, P. D. 2009. Treatment of calf diarrhea: Antimicrobial and ancillary treatments. Vet. Clin. North Am. Food Anim. Pract. 25:101-120. https://doi.org/10.1016/j.cvfa.2008.10.012.

Constable, P. D., P. G. Walker, D. E. Morin, and J. H. Foreman. 1998. Clinical and laboratory assessment of hydration status of neonatal calves with diarrhea. J. Am. Vet. Med. Assoc. 212:991-996.

Dalton, R. G., E. W. Fisher, and W. I. McIntyre. 1965. Changes in blood chemistry, body weight and haemotocrit of calves affected with neonatal diarrhoea. Br. Vet. J. 121:34-42.

Ewaschuk, J. B., J. M. Naylor, M. Chirino-Trejo, and G. A. Zello. 2004. Lactobacillus rhamnosus strain $\mathrm{gg}$ is a potential probiotic for calves. Can. J. Vet. Res. 68:249-253.

Fischer, H., T. E. Machen, J. H. Widdicombe, T. J. Carlson, S. R. King, J. W. Chow, and B. Illek. 2004. A novel extract SB-300 from the stem bark latex of Croton lechleri inhibits CFTR-mediated chloride secretion in human colonic epithelial cells. J. Ethnopharmacol. 93:351-357. https://doi.org/10.1016/j.jep.2004.04.005.

Fisher, E. W., and G. H. De la Fuente. 1972. Water and electrolyte studies in newborn calves with particular reference to the effects of diarrhoea. Res. Vet. Sci. 13:315-322.

Foditsch, C., R. V. Pereira, E. K. Ganda, M. S. Gomez, E. C. Marques, T. Santin, and R. C. Bicalho. 2015. Oral administration of Faecalibacterium prausnitzii decreased the incidence of severe diarrhea and related mortality rate and increased weight gain in preweaned dairy heifers. PLoS One 10:e0145485 https://doi.org/10.1371/ journal.pone.0145485.

Frank, N. A., and J. B. Kaneene. 1993. Management risk factors associated with calf diarrhea in Michigan dairy herds. J. Dairy Sci. 76:1313-1323. https://doi.org/10.3168/jds.S0022-0302(93)774627 .

Garthwaite, B. D., J. K. Drackley, G. C. McCoy, and E. H. Jaster. 1994. Whole milk and oral rehydration solution for calves with diarrhea of spontaneous origin. J. Dairy Sci. 77:835-843.

Groutides, C. P., and A. R. Michell. 1990. Intravenous solutions for fluid therapy in calf diarrhoea. Res. Vet. Sci. 49:292-297.

Gulliksen, S. M., E. Jor, K. I. Lie, I. S. Hamnes, T. Loken, J. Akerstedt, and O. Osteras. 2009. Enteropathogens and risk factors for diarrhea in Norwegian dairy calves. J. Dairy Sci. 92:5057-5066. https://doi.org/10.3168/jds.2009-2080.

Hartmann, H., and S. Reder. 1995. Effects of dehydration on functional parameters of fluid balance as well as effectiveness of rehydration using crystalline or colloidal infusion drips in calves. Tierarztl. Prax. 23:342-350.

Heath, S. E., J. M. Naylor, B. L. Guedo, L. Petrie, C. G. Rousseaux, and O. M. Radostits. 1989. The effects of feeding milk to diarrheic calves supplemented with oral electrolytes. Can. J. Vet. Res. $53: 477-485$.

Heine, J., J. F. Pohlenz, H. W. Moon, and G. N. Woode. 1984. Enteric lesions and diarrhea in gnotobiotic calves monoinfected with cryptosporidium species. J. Infect. Dis. 150:768-775. 
Kaske, M. 1993. Physiological function of the gastrointestinal tract and pathophysiological changes in neonatal diarrhea of calves. Dtsch. Tierarztl. Wochenschr. 100:434-439.

Klein-Jöbstl, D., M. Iwersen, and M. Drillich. 2014. Farm characteristics and calf management practices on dairy farms with and without diarrhea: A case-control study to investigate risk factors for calf diarrhea. J. Dairy Sci. 97:5110-5119. https://doi.org/10.3168/ jds.2013-7695.

Lewis, L. D., and R. W. Phillips. 1978. Pathophysiologic changes due to coronavirus-induced diarrhea in the calf. J. Am. Vet. Med. Assoc. 173:636-642.

Luginbühl, A., K. Reitt, A. Metzler, M. Kollbrunner, L. Corboz, and P. Deplazes. 2005. Field study of the prevalence and diagnosis of diarrhea-causing agents in the newborn calf in a Swiss veterinary practice area. Schweiz. Arch. Tierheilkd. 147:245-252. https://doi. org/10.1024/0036-7281.147.6.245.

Malmuthuge, N., P. J. Griebel, and L. Guan Le. 2015. The gut microbiome and its potential role in the development and function of newborn calf gastrointestinal tract. Front. Vet. Sci. 2:36 https:// doi.org/10.3389/fvets.2015.00036.

O'Handley, R. M., C. Cockwill, T. A. McAllister, M. Jelinski, D. W. Morck, and M. E. Olson. 1999. Duration of naturally acquired giardiosis and cryptosporidiosis in dairy calves and their association with diarrhea. J. Am. Vet. Med. Assoc. 214:391-396.

Oikonomou, G., A. G. Teixeira, C. Foditsch, M. L. Bicalho, V. S. Machado, and R. C. Bicalho. 2013. Fecal microbial diversity in pre-weaned dairy calves as described by pyrosequencing of metagenomic 16s rdna. Associations of faecalibacterium species with health and growth. PLoS One 8:e63157 https://doi.org/10.1371/ journal.pone.0063157.

Phillips, R. W., L. D. Lewis, and K. L. Knox. 1971. Alterations in body water turnover and distribution in neonatal calves with acute diarrhea. Ann. N. Y. Acad. Sci. 176:231-243. https://doi. org/10.1111/j.1749-6632.1971.tb35009.x.

Reynolds, D. J., J. H. Morgan, N. Chanter, P. W. Jones, J. C. Bridger, T. G. Debney, and K. J. Bunch. 1986. Microbiology of calf diarrhoea in southern Britain. Vet. Rec. 119:34-39.

Sen, I., V. Altunok, M. Ok, A. Coskun, and P. D. Constable. 2009. Efficacy of oral rehydration therapy solutions containing sodium bicarbonate or sodium acetate for treatment of calves with naturally acquired diarrhea, moderate dehydration, and strong ion acidosis. J. Am. Vet. Med. Assoc. 234:926-934. https://doi.org/10.2460/ javma.234.7.926.

Smith, D. R. 2012. Field disease diagnostic investigation of neonatal calf diarrhea. Vet. Clin. North Am. Food Anim. Pract. 28:465-481. https://doi.org/10.1016/j.cvfa.2012.07.010.

Smith, G. W. 2009. Treatment of calf diarrhea: Oral fluid therapy. Vet. Clin. North Am. Food Anim. Pract. 25:55-72. https://doi. org/10.1016/j.cvfa.2008.10.006.

Teixeira, A. G., L. Stephens, T. J. Divers, T. Stokol, and R. C. Bicalho. 2015. Effect of crofelemer extract on severity and consistency of experimentally induced enterotoxigenic Escherichia coli diarrhea in newborn Holstein calves. J. Dairy Sci. 98:8035-8043. https:// doi.org/10.3168/jds.2015-9513.

Thiagarajah, J. R., and A. S. Verkman. 2003. Cftr pharmacology and its role in intestinal fluid secretion. Curr. Opin. Pharmacol. 3:594599. https://doi.org/10.1016/j.coph.2003.06.012.

Thiagarajah, J. R., and A. S. Verkman. 2013. Chloride channel-targeted therapy for secretory diarrheas. Curr. Opin. Pharmacol. 13:888-894. https://doi.org/10.1016/j.coph.2013.08.005.

Tradtrantip, L., W. Namkung, and A. S. Verkman. 2010. Crofelemer, an antisecretory antidiarrheal proanthocyanidin oligomer extracted from Croton lechleri, targets two distinct intestinal chloride channels. Mol. Pharmacol. 77:69-78. https://doi.org/10.1124/ mol.109.061051.

Tzipori, S., M. Smith, C. Halpin, T. Makin, and F. Krautil. 1983. Intestinal changes associated with rotavirus and enterotoxigenic Escherichia coli infection in calves. Vet. Microbiol. 8:35-43. https://doi.org/10.1016/0378-1135(83)90017-2.

Tzipori, S. R., T. J. Makin, M. L. Smith, and F. L. Krautil. 1981. Clinical manifestations of diarrhea in calves infected with rotavirus and enterotoxigenic Escherichia coli. J. Clin. Microbiol. 13:1011-1016.

US Department of Agriculture. 2012. Dairy Heifer Raiser, 2011 USDA-APHIS-VS, CEAH, National Animal Health Monitoring System (NAHMS), Fort Collins, CO.

Uyeno, Y., Y. Sekiguchi, and Y. Kamagata. 2010. rRNA-based analysis to monitor succession of faecal bacterial communities in Holstein calves. Lett. Appl. Microbiol. 51:570-577. https://doi. org/10.1111/j.1472-765X.2010.02937.x.

van den Bogaard, A. E., and E. E. Stobberingh. 2000. Epidemiology of resistance to antibiotics. Links between animals and humans. Int. J. Antimicrob. Agents 14:327-335.

Virtala, A. M. K., G. D. Mechor, Y. T. Gröhn, and H. N. Erb. 1996. The effect of calfhood diseases on growth of female dairy calves during the first 3 months of life in New York State. J. Dairy Sci. 79:1040-1049. https://doi.org/10.3168/jds.S0022-0302(96)764573 .

Vlková, E., I. Trojanova, and V. Rada. 2006. Distribution of bifidobacteria in the gastrointestinal tract of calves. Folia Microbiol. (Praha) 51:325-328.

Walker, P. G., P. D. Constable, D. E. Morin, J. K. Drackley, J. H. Foreman, and J. C. Thurmon. 1998. A reliable, practical, and economical protocol for inducing diarrhea and severe dehydration in the neonatal calf. Can. J. Vet. Res. 62:205-213.

Walker, W. L., W. B. Epperson, T. E. Wittum, L. K. Lord, P. J Rajala-Schultz, and J. Lakritz. 2012. Characteristics of dairy calf ranches: Morbidity, mortality, antibiotic use practices, and biosecurity and biocontainment practices. J. Dairy Sci. 95:2204-2214. https://doi.org/10.3168/jds.2011-4727.

Windeyer, M. C., K. E. Leslie, S. M. Godden, D. C. Hodgins, K. D. Lissemore, and S. J. LeBlanc. 2014. Factors associated with morbidity, mortality, and growth of dairy heifer calves up to 3 months of age. Prev. Vet. Med. 113:231-240. https://doi.org/10.1016/j. prevetmed.2013.10.019. 